Bull. Austral. Math. Soc.

$46 \mathrm{E} 30,47 \mathrm{H} 09$

VoL. 68 (2003) [353-369]

\title{
ALMOST CONTRACTIVE RETRACTIONS IN ORLICZ SPACES
}

\section{Grzegorz Lewicki and Giulio Trombetta}

Let $B_{k}$ denote the Euclidean unit ball in $\mathbb{R}^{k}$ equipped with the $k$-dimensional Lebesgue measure and let $\phi: \mathbb{R}^{+} \rightarrow \mathbb{R}^{+}$be a convex function satisfying $\phi(0)$ $=0, \phi(t)>0$ for some $t>0$. Denote by $E_{\phi}=E_{\phi}\left(B_{k}\right)$ the Orlicz space of finite elements (see (1.6)) generated by $\phi$. The aim of this paper is to show that there exists a retraction of the closed unit ball in $E_{\phi}$ onto the unit sphere in $E_{\phi}$ being a $(2+\varepsilon) \gamma_{\phi}$-set contraction (Theorem 3.6), which generalises [9, Corollary $6]$ proved for the case of $L_{p}[-1,1], 1 \leqslant p<\infty$. Here $\gamma_{\phi}$ denote the Hausdorff measure of noncompactness. This theorem is proved both for the Amemiya and the Luxemburg norms. Also some related results concerning the case of $s$-convex $(0<s \leqslant 1)$ functions are presented.

\section{INTRODUCTION}

Let $X$ be a Banach space with the closed unit ball $B$ and the unit sphere $S$. A continuous mapping $R: B \rightarrow S$ is called a retraction if $R x=x$ for any $x \in S$. Let $\psi$ be a measure of noncompactness defined of $X$. A mapping $T: X \supset D(T) \rightarrow X$ is called a $\psi k$-set contraction if there exists $k \geqslant 0$ such that

$$
\psi(T(A)) \leqslant k \psi(A)
$$

for any bounded set $A \subset D(T)$. Set

$$
k_{L}(X)=\inf \{k \geqslant 1: \text { there exists a } k \text {-Lipschizian retraction } R: B \rightarrow S\} .
$$

and

$$
k_{\psi}(X)=\inf \{k \geqslant 1: \text { there exists a }(\psi) \text { k-set contractive retraction } R: B \rightarrow S\} .
$$

By [2] and [6] for any infinite-dimensional Banach space $X, k_{L}(X)<\infty$. By [3], $k_{L}(X) \geqslant 3$ for any Banach space $X$. Also it is easy to see that $k_{\gamma}(X) \leqslant k_{L}(X)$, where $\gamma$ denotes the Hausdorff measure of noncompactness, that is,

$$
\gamma(A)=\inf \left\{r>0: A \subset \bigcup_{i=1}^{k} B\left(x_{i}, r\right), x_{1}, \ldots, x_{k} \in X\right\},
$$

Received 21st November, 2002

Copyright Clearance Centre, Inc. Serial-fee code: 0004-9727/03 \$A2.00+0.00. 
where $A$ is a bounded subset of $X$ and $B\left(x_{i}, r\right)$ denote the closed ball with a centre $x_{i}$ and radius $r$. For more complete information about measures of noncompactness and $(\psi) \mathrm{k}$-sets contractions the reader is referred to $[\mathbf{1}, \mathbf{3}, \mathbf{7}, \mathbf{8}, \mathbf{1 0}]$. Moreover, it has been shown in [10] that $k_{\gamma}\left(C_{\mathbb{R}}[0,1]\right) \leqslant 1$ and in $[9$, Corollary 6$]$ that $k_{\gamma}\left(L_{p}[-1,1]\right) \leqslant 2$.

The aim of this paper is to generalise the above mentionned results to the case of Orlicz spaces (see Theorem 3.6). We consider both the Luxemburg and Amemiya norms (see (1.7) and (1.8)).

Also we prove some results for Orlicz spaces generated by $s$-convex functions. In particular, in $s$-convex case, we introduce a kind of measure of non-compactness $\omega_{\phi}^{s}$ and $\omega_{\phi, A}^{s}$ (see Definition 2.8) which is an analogue of the measure of noncompactness $\omega_{p}$ considered in [9]. We show that in the convex case $(s=1)$

$$
\omega_{\phi}^{1} / 2 \leqslant \gamma_{\phi}^{1} \leqslant \omega_{\phi}^{1} \text { and } \omega_{\phi, A}^{1} / 2 \leqslant \gamma_{\phi, A}^{1} \leqslant \omega_{\phi, A}^{1},
$$

where $\gamma_{\phi}^{1}\left(\gamma_{\phi, A}^{1}\right.$, respectively) is the Hausdorff measure of noncompactness associated with the Luxemburg norm (with the Amemiya norm, respectively), which generalises the classical result concerning $L_{p}$-spaces (see for example, [4]). Also we show that $k_{\omega_{\phi}^{s}}\left(E_{\phi}\right) \leqslant 1$ (Theorem 3.3) and $K_{\omega_{\phi, A}^{s}}\left(E_{\phi}\right) \leqslant 1$, (Theorem 3.4), which generalises [9, Theorem 5].

Now we present some basic facts concerning Orlicz spaces. Let $\phi: \mathbb{R}^{+} \rightarrow \mathbb{R}^{+}$be continuous, $s$-convex function such that $\phi(0)=0$ and $\phi(t)>0$ for some $t>0$. Recall that a function $\phi: \mathbb{R}_{+} \rightarrow \mathbb{R}_{+}$is called $s$-convex for some $0<s \leqslant 1$, if

$$
\phi(a x+b y) \leqslant a^{s} \phi(x)+b^{s} \phi(y)
$$

for $x, y \in \mathbb{R}^{+}, a, b \geqslant 0 a^{s}+b^{s}=1$. Observe that for $s=1$, we get the class of convex functions. Let $(\Omega, \Sigma, \mu)$ be a measure space. Denote by $\mathcal{M}=\mathcal{M}(\Omega, \mathbb{R})$ the set of all real-valued $\mu$-measurable functions defined on $\Omega$. For $f \in \mathcal{M}$ set

$$
\rho_{\phi}(f)=\int_{\Omega} \phi(|f(t)|) d \mu(t) .
$$

By $L_{\phi}=L_{\phi}(\Omega, \Sigma, \mu)$ we denote the Orlicz space generated by $\phi$, that is,

$$
L_{\phi}=\left\{f \in \mathcal{M}: \lim _{\lambda \rightarrow 0} \rho_{\phi}(\lambda f)=0\right\} .
$$

By $E_{\phi}$ we denote the space of finite elements, that is,

$$
E_{\phi}=\left\{f \in \mathcal{M}: \rho_{\phi}(\lambda f)<\infty \text { for any } \lambda>0\right\} .
$$

It is well-known that $E_{\phi}$ is a closed subspace of $L_{\phi}$. Moreover, $L_{\phi}=E_{\phi}$ if and only if the appropriate $\Delta_{2}$ condition holds true (see for example, [5, Theorem 8.14, p. 53]). If 
$\phi$ is an $s$-convex function, we can equip $L_{\phi}$ with an $s$-convex norm (norm if $s=1$ ), given by

$$
\|f\|_{\phi}^{s}=\inf \left\{u>0: \rho_{\phi}\left(f / u^{1 / s}\right) \leqslant 1\right\} .
$$

named the Luxemburg $s$-norm (norm if $s=1$ ).

Observe that, if $\phi(t)=t^{p}$ where $1 \leqslant p<\infty$, then $L_{\phi}=E_{\phi}=L_{p}$ and $\|f\|_{\phi}^{1}$ $=\|f\|_{p}$. If $0<p<1$, then $\phi$ is a $p$-convex function and $\|f\|_{\phi}^{p}=\int_{\Omega}|f(t)|^{p} d \mu(t)$.

It is also well-known, (see for example, [5,Theorem 1.10, p. 6]) that in $s$-convex case, we can consider in $L_{\phi}$ the other $s$-norm (norm if $s=1$ ), called the Amemiya $s$-norm defined by

$$
|f|_{\phi}^{s}=\inf \left\{\left(1+\rho_{\phi}\left(u^{1 / s} f\right)\right) / u: u>0\right\} .
$$

For more detailed information about Orlicz spaces see for example, [5].

\section{TeChNical Lemmas}

Definition 2.1: Let $B_{k}$ denote the unit Euclidean ball in $\mathbb{R}^{k}$. For $x \in \mathbb{R}^{k}$ denote by $\|x\|_{e}$ the Euclidean norm of $x$. Let $\Sigma_{k}$ be the set of all Borel subsets of $B_{k}$ equipped with the Lebesgue measure $\mu_{k}$. Define for $f \in L_{\phi}=L_{\phi}\left(B_{k}, \Sigma_{k}, \mu_{k}\right)\|f\|_{\phi}^{s} \leqslant 1$,

$$
\left(Q_{\phi}^{s} f\right)(t)= \begin{cases}f\left(\frac{2}{1+\|f\|_{\phi}^{s}} t\right) & \text { if }\|t\|_{e} \leqslant \frac{1+\|f\|_{\phi}^{s}}{2} \\ 0 & \text { if }\|t\|_{e}>\frac{1+\|f\|_{\phi}^{s}}{2}\end{cases}
$$

Analogously, for $f \in L_{\phi},|f|_{\phi}^{s} \leqslant 1$, set

$$
\left(Q_{\phi, A}^{s} f\right)(t)= \begin{cases}f\left(\frac{2}{1+|f|_{\phi}^{s}} t\right) & \text { if }\|t\|_{e} \leqslant \frac{1+|f|_{\phi}^{s}}{2} \\ 0 & \text { if }\|t\|_{e}>\frac{1+|f|_{\phi}^{s}}{2}\end{cases}
$$

Lemma 2.2. For any $\lambda>0, f \in L_{\phi}$ with $\|f\|_{\phi}^{s} \leqslant 1$

$$
\rho_{\phi}\left(\lambda Q_{\phi}^{s}(f)\right)=\frac{\left(1+\|f\|_{\phi}^{s}\right)^{k}}{2^{k}} \rho_{\phi}(\lambda f) .
$$

Moreover, for any $b \geqslant 1$,

$$
\rho_{\phi}\left(b \lambda Q_{\phi}^{s}(f)\right) \geqslant b^{s} \frac{\left(1+\|f\|_{\phi}^{s}\right)^{k}}{2^{k}} \rho_{\phi}(\lambda f) .
$$


The same results hold true for the Amemiya $s$-norm $|\cdot|_{\phi}^{s}$.

Proof: Observe that for any $\lambda>0$

$$
\rho_{\phi}\left(\lambda Q_{\phi}^{s}(f)\right)=\int_{\|t\|_{e} \leqslant\left(1+\|f\|_{\phi}^{s}\right) / 2} \phi\left(\lambda\left|f\left(\frac{2}{1+\|f\|_{\phi}^{s}} t\right)\right|\right) d \mu_{k}(t) .
$$

Set $g(t)=\left(2 /\left(1+\|f\|_{\phi}^{s}\right)\right) t$. Note that by changing variables from $t$ to $g(t)$

$$
\begin{aligned}
\rho_{\phi}\left(\lambda Q_{\phi}^{s}(f)\right) & =\left(\frac{1+\|f\|_{\phi}^{s}}{2}\right)^{k} \int_{g^{-1}\left(B_{k}\right)} \phi\left(\lambda\left|f\left(\frac{2}{1+\|f\|_{\phi}^{s}} t\right)\right|\right)\left|\operatorname{det} g^{\prime}(t)\right| d \mu_{k}(t) \\
& =\left(\frac{1+\|f\|_{\phi}^{s}}{2}\right)^{k} \int_{B_{k}} \phi(\lambda|f(s)|) d \mu_{k}(s)=\left(\frac{1+\|f\|_{\phi}^{s}}{2}\right)^{k} \rho_{\phi}(\lambda f)
\end{aligned}
$$

which proves our claim. The same reasoning applies to the operator $Q_{\phi, A}^{s}$ and any function $f \in L_{\phi},|f|_{\phi}^{s} \leqslant 1$.

Now fix $b \geqslant 1$. Then $\phi(b x) \geqslant b^{s} \phi(x)$ for any $x \in \mathbb{R}^{+}$. Hence

$$
\rho_{\phi}\left(b \lambda Q_{\phi}^{s}(f)\right)=\int_{B_{k}} \phi\left(b \lambda\left|f\left(\frac{2}{1+\|f\|_{\phi}^{s}} t\right)\right|\right) d \mu_{k}(t) \geqslant b^{s} \rho_{\phi}\left(Q_{\phi}^{s}(\lambda f)\right) .
$$

By the previous part of the proof,

$$
b^{s} \rho_{\phi}\left(Q_{\phi}^{s}(\lambda f)\right)=b^{s}\left(\frac{1+\|f\|_{\phi}^{s}}{2}\right)^{k} \rho_{\phi}(\lambda f)
$$

which shows (2.2).

Reasoning in the same way, we can show that

$$
\rho_{\phi}\left(b \lambda Q_{\phi, A}^{s}(f)\right) \geqslant b^{s} \frac{\left(1+|f|_{\phi}^{s}\right)^{k}}{2^{k}} \rho_{\phi}(\lambda f)
$$

LEMMA 2.3. For any $f \in L_{\phi},\|f\|_{\phi}^{s} \leqslant 1$,

$$
\left(\frac{1+\|f\|_{\phi}^{s}}{2}\right)^{k}\|f\|_{\phi}^{s} \leqslant\left\|Q_{\phi}^{s}(f)\right\|_{\phi}^{s} \leqslant\|f\|_{\phi}^{s}
$$

The same result holds true for $|\cdot|_{\phi}^{s}$ and $Q_{\phi, A}^{s}$.

Proof: Take any $u>\|f\|_{\phi}^{s}$. By (1.7) and Lemma 2.2 applied to $\lambda=1 / u^{1 / s}$, we get

$$
\rho_{\phi}\left(Q_{\phi}^{s}(f) / u^{1 / s}\right)=\left(\frac{1+\|f\|_{\phi}^{s}}{2}\right)^{k} \rho_{\phi}\left(f / u^{1 / s}\right) \leqslant\left(\frac{1+\|f\|_{\phi}^{s}}{2}\right)^{k} \leqslant 1
$$


Hence $\left\|Q_{\phi}^{s}(f)\right\|_{\phi}^{s} \leqslant\|f\|_{\phi}^{s}$.

Now take any $f \in L_{\phi},|f|_{\phi}^{s} \leqslant 1$. By Lemma 2.2 and (1.8) one can easily show that $\left|Q_{\phi, A}^{s}(f)\right|_{\phi}^{s} \leqslant|f|_{\phi}^{s}$.

Now we prove the second inequality. Let $u=d\left(\left(1+\|f\|_{\phi}^{s}\right) / 2\right)^{k}\|f\|_{\phi}^{s}$ for some $d$ $\epsilon(0,1)$. Then by Lemma 2.2 , and $s$-convexity of $\phi$,

$$
\begin{aligned}
\rho_{\phi}\left(Q_{\phi}^{s}(f) / u^{1 / s}\right) & =\rho_{\phi}\left(\left(\frac{2}{1+\|f\|_{\phi}^{s}}\right)^{k / s} Q_{\phi}^{s}(f) /\left(d\|f\|_{\phi}^{s}\right)^{1 / s}\right) \\
& \geqslant\left(\frac{2}{1+\|f\|_{\phi}^{s}}\right)^{k} \rho_{\phi}\left(Q_{\phi}^{s}(f) /\left(d\|f\|_{\phi}^{s}\right)^{1 / s}\right) \\
& =\rho_{\phi}\left(f /\left(d\|f\|_{\phi}^{s}\right)^{1 / s}\right)>1 .
\end{aligned}
$$

Since $d$ can be an arbitrary number from $(0,1)$,

$$
\left\|Q_{\phi}^{s}(f)\right\|_{\phi}^{s} \geqslant\left(\frac{1+\|f\|_{\phi}^{s}}{2}\right)^{k}\|f\|_{\phi}^{s}
$$

as required.

Finally, we consider the case of $|\cdot|_{\phi}^{s}$. Take any $u>0$. Observe that by Lemma 2.2 ,

$$
\begin{aligned}
\frac{1+\rho_{\phi}\left(u^{1 / s} Q_{\phi, A}^{s}(f)\right)}{u} & =\frac{1+\left(\left(1+|f|_{\phi}^{s}\right) / 2\right)^{k} \rho_{\phi}\left(u^{1 / s} f\right)}{u} \\
& \geqslant\left(\frac{1+|f|_{\phi}^{s}}{2}\right)^{k}\left(\frac{1+\rho_{\phi}\left(u^{1 / s} f\right)}{u}\right) \geqslant\left(\frac{1+|f|_{\phi}^{s}}{2}\right)^{k}|f|_{\phi}^{s} .
\end{aligned}
$$

Hence taking infimum over $u>0$, we get

$$
\left|Q_{\phi, A}^{s}(f)\right|_{\phi}^{s} \geqslant\left(\frac{1+|f|_{\phi}^{s}}{2}\right)^{k}|f|_{\phi}^{s}
$$

which completes the proof.

LemMA 2.4. Let $\left(f_{n}\right)$ be a sequence of functions from $E_{\phi}$ (see (1.6)) and let $f \in E_{\phi},\left\|f_{n}\right\|_{\phi}^{s} \leqslant 1$ for any $n \in \mathbb{N}$ and $\|f\|_{\phi}^{s} \leqslant 1$. If for any $\lambda>0$,

$$
\rho_{\phi}\left(\lambda\left(f_{n}-f\right)\right) \rightarrow 0
$$

then

$$
\rho_{\phi}\left(\lambda\left(Q_{\phi}^{s}\left(f_{n}\right)-Q_{\phi}^{s}(f)\right)\right) \rightarrow 0
$$


for any $\lambda>0$. Analogously, if $\left|f_{n}\right|_{\phi}^{s} \leqslant 1$ for any $n \in \mathbb{N}$ and $|f|_{\phi}^{s} \leqslant 1$, then

$$
\rho_{\phi}\left(\lambda\left(Q_{\phi, A}^{s}\left(f_{n}\right)-Q_{\phi, A}^{s}(f)\right)\right) \rightarrow 0
$$

for any $\lambda>0$.

Proof: Note that by [5, Theorem 6, p. 3],

$$
\rho_{\phi}\left(\lambda\left(f_{n}-f\right)\right) \rightarrow 0
$$

if and only if $\left\|f_{n}-f\right\|_{\phi}^{s} \rightarrow 0$ and

$$
\rho_{\phi}\left(\lambda\left(Q_{\phi}^{s}\left(f_{n}\right)-Q_{\phi}^{s}(f)\right)\right) \rightarrow 0
$$

if and only if $\left\|Q_{\phi}^{s}\left(f_{n}\right)-Q_{\phi}^{s}(f)\right\|_{\phi}^{s} \rightarrow 0$. Observe that

$$
\left\|Q_{\phi}^{s}\left(f_{n}\right)-Q_{\phi}^{s}(f)\right\|_{\phi}^{s} \leqslant\left\|Q_{\phi}^{s}\left(f_{n}\right)-Q_{\phi, n}^{s}(f)\right\|_{\phi}^{s}+\left\|Q_{\phi, n}^{s}(f)-Q_{\phi}^{s}(f)\right\|_{\phi}^{s},
$$

where

$$
\left(Q_{\phi, n}^{s} f\right)(t)= \begin{cases}f\left(\frac{2}{1+\left\|f_{n}\right\|_{\phi}^{s}} t\right) & \text { if }\|t\|_{e} \leqslant \frac{1+\left\|f_{n}\right\|_{\phi}^{s}}{2} \\ 0 & \text { if }\|t\|_{e}>\frac{1+\left\|f_{n}\right\|_{\phi}^{s}}{2}\end{cases}
$$

By Lemma 2.2, for any $\lambda>0$,

$$
\rho_{\phi}\left(\lambda\left(Q_{\phi}^{s}\left(f_{n}\right)-Q_{\phi, n}^{s}(f)\right)\right)=\left(\frac{1+\left\|f_{n}\right\|_{\phi}^{s}}{2}\right)^{k} \rho_{\phi}\left(\lambda\left(f_{n}-f\right)\right) .
$$

Hence $\left\|Q_{\phi}^{s}\left(f_{n}\right)-Q_{\phi, n}^{s}(f)\right\|_{\phi}^{s} \rightarrow 0$. To end the proof, it is sufficient to show that $\left\|Q_{\phi, n}^{s}(f)-Q_{\phi}^{s}(f)\right\|_{\phi}^{s} \rightarrow 0$. To do this, fix $\varepsilon>0$. Since $f \in E_{\phi}$, there exists a continuous function $g: B_{k} \rightarrow \mathbb{R}$, such that $\|g\|_{\phi}^{s}=\|f\|_{\phi}^{s}$ and $\|g-f\|_{\phi}^{s} \leqslant \varepsilon$. Observe that

$$
\begin{aligned}
\left\|Q_{\phi, n}^{s}(f)-Q_{\phi}^{s}(f)\right\|_{\phi}^{s} \leqslant\left\|Q_{\phi, n}^{s}(f)-Q_{\phi, n}^{s}(g)\right\|_{\phi}^{s} \\
\\
+\left\|Q_{\phi, n}^{s}(g)-Q_{\phi}^{s}(g)\right\|_{\phi}^{s}+\left\|Q_{\phi}^{s}(g)-Q_{\phi}^{s}(f)\right\|_{\phi}^{s}
\end{aligned}
$$

Note that by Lemma 2.2 , for any $\lambda>0$ and $n \in \mathbb{N}$,

$$
\rho_{\phi}\left(\lambda\left(Q_{\phi, n}^{s}(f)-Q_{\phi, n}^{s}(g)\right)\right)=\left(\frac{1+\left\|f_{n}\right\|_{\phi}^{s}}{2}\right)^{k} \rho_{\phi}(\lambda(f-g)) .
$$

Hence, by (1.7),

$$
\left\|Q_{\phi, n}^{s}(f)-Q_{\phi, n}^{s}(g)\right\|_{\phi}^{s} \leqslant\|f-g\|_{\phi}^{s} \leqslant \varepsilon
$$


Analogously, since $\|f\|_{\phi}^{s}=\|g\|_{\phi}^{s}$,

$$
\left\|Q_{\phi}^{s}(f)-Q_{\phi}^{s}(g)\right\|_{\phi}^{s} \leqslant\|f-g\|_{\phi}^{s} \leqslant \varepsilon .
$$

Now we show that

$$
\left\|Q_{\phi, n}^{s}(g)-Q_{\phi}^{s}(g)\right\|_{\phi}^{s} \rightarrow 0 .
$$

Let $\|g\|_{\infty}$ denote the supremum norm of $g$. Fix $\lambda>0$ and set

$$
D_{n}=\left\{t \in S_{k}: \frac{1+\|f\|_{\phi}^{s}}{2} \leqslant\|t\|_{e} \leqslant \frac{1+\left\|f_{n}\right\|_{\phi}^{s}}{2}\right\}
$$

If $\left\|f_{n}\right\|_{\phi}^{s} \geqslant\|f\|_{\phi}^{s}$, then by Lemma 2.2 ,

$$
\begin{aligned}
\int_{B_{k}} \phi & \left(\lambda\left|\left(Q_{\phi, n}^{s}(g)\right)-\left(Q_{\phi}^{s}(g)\right)(t)\right|\right) d \mu_{k}(t) \\
= & \int_{\|t\|_{e} \leqslant \frac{1+\|f\|_{\phi}^{s}}{2} \phi\left(\lambda \mid g\left(\frac{2}{1+\left\|f_{n}\right\|_{\phi}^{s}} t\right)\right.} \\
& \left.\quad-g\left(\frac{2}{1+\|f\|_{\phi}^{s}} t\right) \mid\right) d \mu_{k}(t)+\int_{D_{n}} \phi\left(\lambda\left|g\left(\frac{2}{1+\left\|f_{n}\right\|_{\phi}^{s}} t\right) d \mu_{k}(t)\right|\right) \\
& \leqslant\left(\frac{1+\|f\|_{\phi}^{s}}{2}\right)^{k} \int_{B_{k}} \phi\left(\lambda\left|g\left(\frac{1+\|f\|_{\phi}^{s}}{1+\left\|f_{n}\right\|_{\phi}^{s}} t\right)-g(t)\right|\right) d \mu_{k}(t)+\mu_{k}\left(D_{n}\right) \phi\left(\lambda\|g\|_{\infty}\right) .
\end{aligned}
$$

Analogously, if $\left\|f_{n}\right\|_{\phi}^{s}<\|f\|_{\phi}^{s}$, then

$$
\begin{aligned}
& \int_{B_{k}} \phi\left(\lambda\left|\left(Q_{\phi, n}^{s}(g)\right)-\left(Q_{\phi}^{s}(g)\right)(t)\right|\right) d \mu_{k}(t) \\
\leqslant & \left(\frac{1+\left\|f_{n}\right\|_{\phi}^{s}}{2}\right)^{k} \int_{B_{k}} \phi\left(\lambda\left|g\left(\frac{1+\left\|f_{n}\right\|_{\phi}^{s}}{1+\|f\|_{\phi}^{s}} t\right)-g(t)\right|\right) d \mu_{k}(t)+\mu_{k}\left(C_{n}\right) \phi\left(\lambda\|g\|_{\infty}\right),
\end{aligned}
$$

where

$$
C_{n}=\left\{t \in S_{k}: \frac{1+\left\|f_{n}\right\|_{\phi}^{s}}{2} \leqslant\|t\|_{e} \leqslant \frac{1+\|f\|_{\phi}^{s}}{2}\right\}
$$

Set for $n \in \mathbb{N}$

$$
h_{n}(t)= \begin{cases}\phi\left(\lambda\left|g\left(\frac{1+\|f\|_{\phi}^{s}}{1+\left\|f_{n}\right\|_{\phi}^{s}}(t)\right)-g(t)\right|\right) & \text { if }\left\|f_{n}\right\|_{\phi}^{s} \geqslant\|f\|_{\phi}^{s} \\ \phi\left(\lambda\left|g\left(\frac{1+\left\|f_{n}\right\|_{\phi}^{s}}{1+\|f\|_{\phi}^{s}}(t)\right)-g(t)\right|\right) & \text { if }\left\|f_{n}\right\|_{\phi}^{s}<\|f\|_{\phi}^{s}\end{cases}
$$


Note that by the continuity of $g, h_{n}(t) \rightarrow 0$ for any $t \in B_{k}$. Moreover, $\left|h_{n}(t)\right|$ $\leqslant \phi\left(2 \lambda\|g\|_{\infty}\right)$ for any $t \in B_{k}$. Since $\mu_{k}\left(B_{k}\right)<\infty$, by the Lebesgue dominated convergence theorem

$$
\int_{B_{k}} h_{n}(t) d \mu_{k}(t) \rightarrow 0
$$

Since, $\mu_{k}\left(C_{n}\right) \rightarrow 0$ and $\mu_{k}\left(D_{n}\right) \rightarrow 0$, by (2.3) $-(2.5)$, for any $\lambda>0$,

$$
\rho_{\phi}\left(\lambda\left(Q_{\phi, n}^{s}(g)-Q_{\phi}^{s}(g)\right)\right) \rightarrow 0
$$

and consequently $\left\|Q_{\phi, n}^{s}(g)-Q_{\phi}^{s}(g)\right\|_{\phi}^{s} \rightarrow 0$.

Reasoning in the same way, we can show that for any $\lambda>0$,

$$
\rho_{\phi}\left(\lambda\left(Q_{\phi, A}^{s}\left(f_{n}\right)-Q_{\phi, A}^{s}(f)\right)\right) \rightarrow 0
$$

The proof is complete.

By Lemma 2.4 and [5, Theorem 6, p. 3] one can easily deduce

Corollary 2.5. Let $f_{n}, f \in E_{\phi},\left\|f_{n}\right\|_{\phi}^{s} \leqslant 1$ for any $n \in \mathbb{N}$ and $\|f\|_{\phi}^{s} \leqslant 1$. If $\left\|f_{n}-f\right\|_{\phi}^{s} \rightarrow 0$ then

$$
\left\|Q_{\phi}^{s}\left(f_{n}\right)-Q_{\phi}^{s}(f)\right\|_{\phi}^{s} \rightarrow 0 .
$$

Analogously if, $\left|f_{n}\right|_{\phi}^{s} \leqslant 1$ for any $n \in \mathbb{N},|f|_{\phi}^{s} \leqslant 1$ and $\left|f_{n}-f\right|_{\phi}^{s} \rightarrow 0$ then

$$
\left|Q_{\phi, A}^{s}\left(f_{n}\right)-Q_{\phi, A}^{s}(f)\right|_{\phi}^{s} \rightarrow 0
$$

DEFINITION 2.6: Let $f \in L_{\phi}\left(B_{k}\right) \cap L_{1}\left(B_{k}\right)$. Set for $r>0, B_{k}(r)=\left\{t \in \mathbb{R}^{k}\right.$ : $\left.\|t\|_{e} \leqslant r\right\}$. For any $h>0$, by $f_{h}$ we denote the Steklov function of $f$, that is

$$
f_{h}(t)=\left(\int_{t+B_{k}(h)} f(u) d \mu_{k}(u)\right) / \mu_{k}\left(B_{k}(h)\right)
$$

REMARK 2.7. Observe that if $f \in L_{1}\left(B_{k}\right)$, then $f_{h}$ is well-defined and continuous for any $h>0$. Hence $f_{h} \in E_{\phi}\left(B_{k}\right)$. Moreover, if $\phi$ is a convex function then $L_{\phi}\left(B_{k}\right)$ $\subset L_{1}\left(B_{k}\right)$, (as a subset) since $\mu_{k}\left(B_{k}\right)<\infty$. Hence in this case $f_{h} \in E_{\phi}$ for any $h>0$ and $f \in L_{\phi}$.

DEFINITION 2.8: Let $\phi$ be an $s$-convex function and let $B \subset L_{\phi}\left(B_{k}\right) \cap L_{1}\left(B_{k}\right)$ be a bounded set with respect to the Luxemburg $s$-convex norm $\|\cdot\|_{\phi}^{s}$. Set

$$
\omega_{\phi}^{s}(B)=\lim _{\delta \rightarrow 0}\left(\sup _{f \in B} \sup _{0<h \leqslant \delta}\left\|f-f_{h}\right\|_{\phi, \mathbb{R}^{k}}^{s}\right)
$$


where $\|\cdot\|_{\phi, \mathbb{R}^{k}}^{s}$ denotes the $s$-convex Luxemburg norm associated with a modular

$$
\rho_{\phi, \mathbb{R}^{k}}(f)=\int_{\mathbb{R}^{k}} \phi(|f(t)|) d \mu_{k}(t) .
$$

In the case of the Amemiya $s$-norm $|\cdot|_{\phi}^{s}($ see (1.8)) we put

$$
\omega_{\phi, A}^{s}(B)=\lim _{\delta \rightarrow 0}\left(\sup _{f \in B} \sup _{0<h \leqslant \delta}\left|f-f_{h}\right|_{\phi, \mathbb{R}^{k}}^{s}\right) .
$$

PROPOSITION 2.9. Let $\phi$ be an $s$-convex function such that

$$
\phi^{*}(v)=\sup _{u>0}(u v-\phi(u))
$$

is finite for any $v \geqslant 0$. Let $\gamma_{\phi}^{s}\left(\gamma_{\phi, A}^{s}\right.$ respectively) denote the Hausdorff measure of noncompactness in $L_{\phi}\left(B_{k}, \Sigma_{k}, \mu_{k}\right)$ with respect to the Luxemburg s-norm (to the Amemiya s-norm respectively). Let $B \subset E_{\phi}$ (see (1.6)) be a bounded set. Then

$$
\gamma_{\phi}^{s}(B) \leqslant \omega_{\phi}^{s}(B)
$$

and

$$
\gamma_{\phi, A}^{s}(B) \leqslant \omega_{\phi, A}^{s}(B)
$$

Moreover, if $s=1$, that is, $\phi$ is a convex function, then

$$
\gamma_{\phi}^{1}(B) \geqslant \frac{\omega_{\phi}^{1}(B)}{2}
$$

and

$$
\gamma_{\phi, A}^{1}(B) \geqslant \frac{\omega_{\phi, A}^{1}(B)}{2}
$$

Proof: First we consider the case of the Luxemburg $s$-norm. Since $\phi^{*}(v)<+\infty$, for any $v \geqslant 0$, by (2.6),

$$
|f(t)| / u^{1 / s} \leqslant \phi\left(\left|f(t) / u^{1 / s}\right|\right)+\phi^{*}(1),
$$

for any $f \in L_{\phi}, t \in B_{k}$ and $u>\|f\|_{\phi}^{s}$. By integrating the above inequality, and (1.4) we easily get that $f \in L_{1}\left(B_{k}\right)$. Hence by Remark $2.7, \omega_{\phi}^{s}(B)$ is well-defined for any bounded set $B \subset E_{\phi}\left(B_{k}\right)$ (see Definition 2.8).

Now fix $b>\omega_{\phi}^{s}(B)$ and take $\varepsilon \in\left(0,\left(b-\omega_{\phi}^{s}(B)\right) / 2\right)$. Then there exists $\delta>0$ such that $0<h<\delta$,

$$
\sup _{f \in B}\left(\left\|f-f_{h}\right\|_{\phi, \mathbb{R}^{k}}^{s}\right)<b-\varepsilon
$$


Fix $: 0<h<\delta$ and let $S_{h}=\left\{f_{h}: f \in B\right\}$. Observe that by (2.6), for any $k \in \mathbb{N}$ and $u>0$,

$$
u \leqslant \phi(u) / k+\phi^{*}(k) / k .
$$

Since $\phi^{*}(k)<+\infty$ for any $k \in \mathbb{N}$, reasoning as in [5, Theorem 9.11, p. 61], we can show that $S_{h}$ is a conditionaly compact set in $E_{\phi}$. Hence there exist $g_{1}, \ldots, g_{\mathfrak{n}(\varepsilon)} \in E_{\phi}$ such that for any $f \in B,\left\|f_{h}-g_{i}\right\|_{\phi}^{s} \leqslant \varepsilon$ for some $i \in\{1, \ldots, n(\varepsilon)\}$. Hence by the triangle inequality for any $f \in B$ there exists $i \in\{1, \ldots, n(\varepsilon)\}$ such that

$$
\left\|f-g_{i}\right\|_{\phi}^{s} \leqslant\left\|f-f_{h}\right\|_{\phi}^{s}+\left\|f_{h}-g_{i}\right\|_{\phi}^{s} \leqslant b .
$$

Hence $\gamma_{\phi}^{s}(B) \leqslant b$ and consequently, $\gamma_{\phi}^{s}(B) \leqslant \omega_{\phi}^{s}(B)$, as required.

The same reasoning applies to the case of the Amemiya norm.

To prove the second part, assume that $\phi$ is a convex function. By $[5$, Theorem 9.10, p. 61] for any $f \in L_{\phi}\left(B_{k}\right)$, and $h>0$,

$$
\left\|f_{h}\right\|_{\phi, \mathbb{R}^{k}}^{1} \leqslant\|f\|_{\phi, \mathbb{R}^{k}}^{1}=\|f\|_{\phi}^{1}
$$

Fix $b>\gamma_{\phi}^{1}(B)$ and take $\varepsilon \in\left(0,\left(b-\gamma_{\phi}^{1}(B)\right) / 2\right)$. Then we can find $g_{1}, \ldots, g_{n(b)}$ $\in E_{\phi}\left(B_{k}\right)$ such that for any $f \in B,\left\|f-g_{i}\right\|_{\phi}^{1} \leqslant b-\varepsilon$, for some $i \in\{1, \ldots, n(b)\}$. Since $B \subset E_{\phi}$, we can assume that $g_{i}$ are continuous functions. Since $B_{k}$ is a compact set, there exists $\delta>0$ such that for any $h<\delta$ and $i=1, \ldots, n(b)$

$$
\left\|g_{i}-\left(g_{i}\right)_{h}\right\|_{\phi, \mathbb{R}^{k}}^{1} \leqslant \varepsilon
$$

Hence, by (2.7) and (2.8), for any $f \in B$ and $0<h<\delta$

$$
\begin{aligned}
\left(\left\|f-f_{h}\right\|_{\phi, \mathbb{R}^{k}}^{1}\right) / 2 & \leqslant\left(\left\|f-g_{i}\right\|_{\phi, \mathbb{R}^{k}}^{1}+\left\|f_{h}-\left(g_{i}\right)_{h}\right\|_{\phi, \mathbb{R}^{k}}^{1}+\left\|g_{i}-\left(g_{i}\right)_{h}\right\|_{\phi, \mathbb{R}^{k}}^{1}\right) / 2 \\
& \leqslant\left\|f-g_{i}\right\|_{\phi}^{1}++\left(\left\|g_{i}-\left(g_{i}\right)_{h}\right\|_{\phi, \mathbb{R}^{k}}^{1}\right) / 2 \leqslant b-\varepsilon+\varepsilon / 2<b .
\end{aligned}
$$

Consequently

$$
\gamma_{\phi}^{1}(B) \geqslant \frac{\omega_{\phi}^{1}(B)}{2}
$$

as required. The same reasoning applies to the case of the Amemiya norm.

Proposition 2.10. For any $s$-convex function $\phi$, satisfying the assumptions of Proposition 2.9, $\lambda \in \mathbb{R}$ and bounded subsets $C, D \subset E_{\phi}$ the following conditions are satisfied:

$$
\begin{aligned}
\omega_{\phi}^{s}(C) & =0 \text { implies } C \text { is conditionally compact } \\
\omega_{\phi}^{s}\left(\mathrm{cl}^{s}(C)\right) & =\omega_{\phi}^{s}(C)
\end{aligned}
$$


where

$$
\operatorname{cl}^{s}(C)=\mathrm{cl}\left(C^{s}=\left\{f \in E_{\phi}: f=\sum_{j=1}^{k} a_{j} f_{j}: f_{j} \in C, a_{1}, \ldots ; a_{k} \geqslant 0, \sum_{j=1}^{k} a_{j}^{s}=1\right\}\right)
$$

$$
\begin{aligned}
\omega_{\phi}^{s}(C \cup D) & =\max \left\{\omega_{\phi}^{s}(C), \omega_{\phi}^{s}(D)\right\} ; \\
\omega_{\phi}^{s}(C+D) & \leqslant \omega_{\phi}^{s}(C)+\omega_{\phi}^{s}(D) ; \\
\omega_{\phi}^{s}(\lambda C) & =|\lambda|^{s} \omega_{\phi}^{s}(C) .
\end{aligned}
$$

Moreover, if $\phi$ is convex, then $\omega_{\phi}^{1}(C)=0$ for any conditionally compact set $C$. The same properties holds true for $\omega_{\phi, A}^{\mathbf{s}}$.

Proof: Suppose that $\omega_{\phi}^{s}(C)=0$. Then by Proposition 2.9, $\gamma_{\phi}^{s}(C)=0$ and consequently $\mathrm{cl}(C)$ is a compact set. If $\phi$ is convex, then again by Proposition 2.9 $\omega_{\phi}^{1}(C)=0$ for any conditionally compact set $C$.

To prove (2.10), first we show that for any fixed $h>0$, if $\left\|f_{n}\right\|_{\phi, \mathbb{R}^{k}}^{s} \rightarrow 0$, then $\left\|\left(f_{n}\right)_{h}\right\|_{\phi, \mathbb{R}^{k}}^{s} \rightarrow 0$. Suppose, this is not true. Without loss of generality, we can assume $\left\|f_{n}\right\|_{\phi, \mathbb{R}^{k}}^{s} \rightarrow 0$, and $\left\|\left(f_{n}\right)_{h}\right\|_{\phi, \mathbb{R}^{k}}^{s} \geqslant d>0$. By our assumptions, reasoning as in [5, Theorem 9.11, p. 61$]$, we can show that the sequence $\left\{\left(f_{n}\right)_{h}\right\}$ contains a subsequence (we denote it again by $\left.\left(f_{n}\right)_{h}\right)$, tending uniformly on $\mathbb{R}^{k}$ to a function $g$ continuous on $B_{k}(1+h)$ and equal 0 outside this set. By our assumptions on $\left\{\left(f_{n}\right)_{h}\right\}, g(t) \neq 0$ for some $t \in B_{k}(1+h)$. Observe that

$$
\mu_{k}\left(C_{n}=\left\{s \in B_{k}(h):\left|f_{n}(s+t)-g(t)\right| \geqslant|g(t)| / 2\right\}\right) \rightarrow 0 .
$$

Consequently, $\mu_{k}\left(B_{k}(h) \backslash C_{n}\right) \rightarrow \mu_{k}\left(B_{h}\right)$. Note that

$$
B_{k}(h) \backslash C_{n} \subset\left\{s \in \mathbb{R}^{k}:\left|f_{n}(s+t)\right| \geqslant|g(t)| / 2\right\} .
$$

Hence $\left\{f_{n}\right\}$ does not converge to 0 in measure. By $\left[\mathbf{5}\right.$, Lemma 9.2, p. 56], $\left\|f_{n}\right\|_{\phi, \mathbb{R}^{k}}^{s}$ does not converge to 0 , a contradiction.

By the previous part of the proof, we get immediately for any bounded set $C \subset E_{\phi}$

$$
\omega_{\phi}^{s}\left(\mathrm{cl}^{s}(C)\right)=\omega_{\phi}^{s}(C)
$$

Now take any $f \in C^{s}, f=\sum_{j=1}^{n} a_{j} f_{j}$, where for $j=1, \ldots, n f_{j} \in C, a_{j} \geqslant 0, \sum_{j=1}^{n} a_{j}^{s}=\cdot 1$. Observe that for any $h>0$,

$$
\left\|f-f_{h}\right\|_{\phi, \mathbf{R}^{k}}^{s} \leqslant \sum_{j=1}^{n} a_{j}^{s}\left\|f_{j}-\left(f_{j}\right)_{h}\right\|_{\phi, \mathbf{R}^{k}}^{s} \leqslant \max _{j=1, \ldots, n}\left\|f_{j}-\left(f_{j}\right)_{h}\right\|_{\phi, \mathbf{R}^{k}}^{s}
$$


Consequently,

$$
\omega_{\phi}^{s}\left(\operatorname{cl}\left(C^{s}\right)\right)=\omega_{\phi}^{s}\left(C^{s}\right) \leqslant \omega_{\phi}^{s}(C)
$$

Since the opposite inequality is obvious, (2.10) is proved. The other properties follow immediately from Definition 2.8.

The same proof applies to the case of $\omega_{\phi, A}^{s}$.

LEMMA 2.11. For any $f \in L_{\phi}\left(B_{k}\right) \cap L_{1}\left(B_{k}\right),\|f\|_{\phi}^{s}=\|f\|_{\phi, \mathbb{R}^{k}}^{s} \leqslant 1$, (we put $f(t)=0$ for $\left.\|t\|_{e}>1\right), \lambda, h>0$

$\int_{\mathbb{R}^{k}}\left(\phi\left(\lambda\left|\left(Q_{\phi}^{s} f\right)(t)-\left(Q_{\phi}^{s} f\right)_{h}(t)\right|\right) d \mu_{k}(t)\right)=\left(\frac{1+\|f\|_{\phi}^{s}}{2}\right)^{k} \int_{\mathbb{R}^{k}} \phi\left(\lambda\left|\left(f-f_{h / a}\right)(t)\right|\right) d \mu_{k}(t)$

where $a=\left(\left(1+\|f\|_{\phi}^{s}\right) / 2\right)$. The same result holds true for any $f \in L_{\phi}\left(B_{k}\right),|f|_{\phi}^{s} \leqslant 1$ and $Q_{\phi, A}^{s}$ with $a=\left(\left(1+|f|_{\phi}^{s}\right) / 2\right)$.

Proof: Fix $f \in L_{\phi},\|f\|_{\phi}^{s} \leqslant 1$ and $\lambda>0$. Set for any $t \in R^{k}, u(t)=t / a$. Then after changing variables from $t$ to $u$, we get

$$
\begin{aligned}
\int_{\mathbb{R}^{k}} \phi\left(\lambda\left|\left(Q_{\phi}^{s} f\right)(t)-\left(Q_{\phi}^{s} f\right)_{h}(t)\right| d \mu_{k}(t)\right) & =a^{k} \int_{\mathbb{R}^{k}} \phi\left(\lambda\left|\left(Q_{\phi}^{s} f\right)(a u)-\left(Q_{\phi}^{s} f\right)_{h}(a u)\right| d \mu_{k}(u)\right) \\
& =a^{k} \int_{\mathbb{R}^{k}} \phi\left(\lambda\left|f(u)-\left(Q_{\phi}^{s} f\right)_{h}(a u)\right| d \mu_{k}(u)\right) .
\end{aligned}
$$

To end the proof of the lemma, we show that $\left(Q_{\phi}^{s} f\right)_{h}(a u)=f_{h / a}(u)$. Observe that

$$
\left(Q_{\phi}^{s} f\right)_{h}(a u)=\left(\int_{a u+B_{k}(h)} f(s / a) \chi_{B_{k}}(s / a) d \mu_{k}(s)\right) / \mu_{k}\left(B_{k}(h)\right)
$$

where $\chi_{B_{k}}$ denotes the characteristic function of $B_{k}$. Set $z(s)=s / a$. After changing variables from $s$ to $z$ we get

$$
\begin{aligned}
\left(Q_{\phi}^{s} f\right)_{h}(a u) & =a^{k}\left(\int_{u+B_{k}(h / a)} f(z) \chi B_{k}(z) d \mu_{k}(z)\right) / \mu_{k}\left(B_{k}(h)\right) \\
& =\left(\int_{u+B_{k}(h / a)} f(z) \chi_{B_{k}}(z) d \mu_{k}(z)\right) / \mu_{k}\left(B_{k}(h / a)\right)=\left(f_{h / a}\right)(u) .
\end{aligned}
$$

The same reasoning applies to the Amemiya $s$-norm. The proof is complete.

Applying Lemma 2.11 and the definitions of the Luxemburg and the Amemiya $s$-norms one can easily get 
Corollary 2.12. For any $f \in L_{\phi} \cap L_{1}\left(B_{k}\right),\|f\|_{\phi}^{s}=\|f\|_{\phi, \mathbf{R}^{k}}^{s} \leqslant 1$,

$$
\left\|Q_{\phi}^{s}(f)-\left(Q_{\phi}^{s} f\right)_{h}\right\|_{\phi, \mathbb{R}^{k}}^{s} \leqslant\left\|f-f_{h / a}\right\|_{\phi, \mathbf{R}^{k}}^{s}
$$

If $|f|_{\phi}^{s} \leqslant 1$, then

$$
\left|Q_{\phi, A}^{s}(f)-\left(Q_{\phi, A}^{s} f\right)_{h}\right|_{\phi, \mathbb{R}^{k}}^{s} \leqslant\left|f-f_{h / a}\right|_{\phi, \mathbb{R}^{k}}^{s} .
$$

THEOREM 2.13. For any set $B \subset\left\{f \in L_{\phi} \cap L_{1}\left(B_{k}\right):\|f\|_{\phi}^{s} \leqslant 1\right\}$,

$$
\omega_{\phi}^{s}\left(Q_{\phi}^{s}(B)\right) \leqslant \omega_{\phi}^{s}(B)
$$

and for any set $B \subset\left\{f \in L_{\phi} \cap L_{1}\left(B_{k}\right):|f|_{\phi}^{s} \leqslant 1\right\}$,

$$
\omega_{\phi, A}^{s}\left(Q_{\phi, A}^{s}(B)\right) \leqslant \omega_{\phi, A}^{s}(B) .
$$

Proof: Follows immediately from Definition 2.8 and Corollary 2.12 .

\section{MAIN RESULTS.}

Let $B_{\phi}\left(B_{\phi, A}\right.$ respectively) denote the unit ball in $E_{\phi}=E_{\phi}\left(B_{k}, \Sigma_{k}, \mu_{k}\right)$ (see (1.6)) with respect to the Luxemburg $s$-norm (with respect to the Amemiya $s$-norm respectively). For any $u>0$ define $P_{\phi, u}: B_{\phi} \rightarrow E_{\phi}$ by

$$
\left(P_{\phi, u} f\right)(t)=\max \left\{0, u\left(2\|t\|_{e}-\|f\|_{\phi}^{s}-1\right)\right\} .
$$

Analogously, for any $u>0$ define $P_{\phi, u, A}: B_{\phi, A} \rightarrow E_{\phi}$ by

$$
\left(P_{\phi, u, A} f\right)(t)=\max \left\{0, u\left(2\|t\|_{e}-|f|_{\phi}^{s}-1\right)\right\} .
$$

Set

$$
T_{\phi, u}(f)=Q_{\phi}^{s}(f)+P_{\phi, u}(f)
$$

and

$$
T_{\phi, u, A}(f)=Q_{\phi, A}^{s}(f)+P_{\phi, u, A}(f)
$$

where $Q_{\phi}^{s}$ and $Q_{\phi, A}^{s}$ are given by Definition 2.1. Observe that $\left(Q_{\phi}^{s} f\right)(t)=0$ if and only if $\|t\|_{e}>\left(1+\|f\|_{\phi}^{s}\right) / 2$ and for any $u>0\left(P_{\phi, u}^{f}\right)(t)=0$ if and only if $\|t\|_{e}$ $\leqslant\left(1+\|f\|_{\phi}^{s}\right) / 2$. Hence for any $u, \lambda>0$ and $f \in B_{\phi}$

$$
\rho_{\phi}\left(\lambda\left(Q_{\phi}^{s} f\right)\right) \leqslant \rho_{\phi}\left(\lambda\left(T_{\phi, u} f\right)\right)
$$


Consequently, for any $u>0, f \in B_{\phi}$

$$
\left\|Q_{\phi}^{s} f\right\|_{\phi}^{s} \leqslant\left\|T_{\phi, u} f\right\|_{\phi}^{s} .
$$

Analogously, for any $u>0, f \in B_{\phi, A}$

$$
\left|Q_{\phi, A}^{s} f\right|_{\phi}^{s} \leqslant\left|T_{\phi, u, A} f\right|_{\phi}^{s} .
$$

Observe that for any $u>0, f \in B_{\phi}$ and $z, t \in B_{k}$,

$$
\left|P_{\phi, u} f(t)-P_{\phi, u} f(z)\right| \leqslant 2 u\|t-z\|_{e} .
$$

Consequently for any $\varepsilon>0$ there exist $\delta>0$ such that for any $f \in B_{\phi}$ and $0<h<\delta$,

$$
\left\|P_{\phi, u} f-\left(P_{\phi, u} f\right)_{h}\right\|_{\infty}=\sup _{t \in B_{k}}\left(\left|P_{\phi, u} f(t)-\left(P_{\phi, u} f\right)_{h}(t)\right|\right) \leqslant \varepsilon .
$$

This implies immediately that for any $B \subset E_{\phi} \cap L_{1}\left(B_{k}\right), \omega_{\phi}^{s}\left(P_{\phi, u}(B)\right)=0$. Hence by Proposition 2.10 and Theorem 2.13, for any $B \subset B_{\phi} \cap L_{1}\left(B_{k}\right)$

$$
\omega_{\phi}^{s}\left(T_{\phi, u}(B)\right) \leqslant \omega_{\phi}^{s}\left(Q_{\phi}^{s}(B)\right)+\omega_{\phi}^{s}\left(P_{\phi, u}(B)\right) \leqslant \omega_{\phi}^{s}(B) .
$$

Analogously, for any $B \subset B_{\phi, A} \cap L_{1}\left(B_{k}\right)$,

$$
\omega_{\phi, A}^{s}\left(T_{\phi, u, A}(B)\right) \leqslant \omega_{\phi, A}^{s}\left(Q_{\phi, A}^{s}(B)\right)+\omega_{\phi, A}^{s}\left(P_{\phi, u, A}(B)\right) \leqslant \omega_{\phi, A}^{s}(B) .
$$

LEMMA 3.1. For any $\varepsilon>0$ there esists $u_{\varepsilon}>0$ such that for any $f \in B_{\phi}$, $\left\|T_{\phi, u_{\varepsilon}}\right\|_{\phi}^{s} \geqslant 1-\varepsilon$.

Proof: Fix $\varepsilon>0$. Choose $\delta>0$ such that

$$
\frac{(1-\delta)(2-\delta)^{k}}{2^{k}}>1-\varepsilon
$$

If $\|f\|_{\phi}^{s} \geqslant 1-\delta$, then by Lemma 2.3 and (3.5), for any $u>0$

$$
\left\|T_{\phi, u} f\right\|_{\phi}^{s} \geqslant\left\|Q_{\phi}^{s} f\right\|_{\phi}^{s} \geqslant\|f\|_{\phi}^{s}\left(\left(1+\|f\|_{\phi}^{s}\right) / 2\right)^{k} \geqslant \frac{(1-\delta)(2-\delta)^{k}}{2^{k}}>1-\varepsilon .
$$

Now suppose $\|f\|_{\phi}^{s}<1-\delta$. Since $\operatorname{supp}\left(Q_{\phi}^{s} f\right) \cap \operatorname{supp}\left(P_{\phi, u}^{s} f\right)=\emptyset$ for any $u>0$,

$$
\begin{aligned}
\rho_{\phi}\left(T_{\phi, u} f\right) & =\rho_{\phi}\left(Q_{\phi}^{s} f\right)+\rho_{\phi}\left(P_{\phi, u} f\right) \\
& \geqslant \rho_{\phi}\left(Q_{\phi}^{s} f\right)+\int_{B_{k} \backslash B_{k}(1-\delta / 4)} \phi\left(u\left(2\|t\|_{e}-\|f\|_{\phi}^{s}-1\right)\right) d \mu_{k}(t) \\
& \geqslant \rho_{\phi}\left(Q_{\phi}^{s} f\right)+\mu_{k}\left(B_{k} \backslash B_{k}(1-\delta / 4)\right) \phi(u(2-\delta / 2-(1-\delta)-1)) \\
& =\rho_{\phi}\left(Q_{\phi}^{s} f\right)+\mu_{k}\left(B_{k} \backslash B_{k}(1-\delta / 4)\right) \phi(u \delta / 2) .
\end{aligned}
$$


Hence, we can find $u_{\varepsilon}>0$ such that for any $f \in B_{\phi},\|f\|_{\phi}^{s}<1-\delta, \rho_{\phi}\left(T_{\phi, u} f\right)>1$ and consequently,

$$
\left\|\left(T_{\phi, u} f\right)\right\|_{\phi}^{s} \geqslant 1>1-\varepsilon,
$$

which completes the proof.

LEMma 3.2. For any $\varepsilon>0$ there exists $u_{\varepsilon}>0$ such that for any $f \in B_{\phi, A}$, $\left|T_{\phi, u_{\varepsilon}, A}\right|_{\phi}^{s} \geqslant 1-\varepsilon$.

ProOF: Fix $\varepsilon>0$. Choose $\delta>0$ such that

$$
\frac{(1-\delta)(2-\delta)^{k}}{2^{k}}>1-\varepsilon
$$

If $|f|_{\phi}^{s} \geqslant 1-\delta$, then reasoning as in Lemma 3.1 , we get $\left|T_{\phi, u, A}\right|_{\phi}^{s}>1-\varepsilon$ for any $u>0$. So assume $|f|_{\phi}^{s}<1-\delta$. Fix $k>0$. If $k \leqslant 1$, then

$$
\frac{1+\rho_{\phi}\left(k^{1 / s} T_{\phi, u, A} f\right)}{k} \geqslant 1 / k \geqslant 1 .
$$

for any $u>0$. If $k>1$, then by $s$-convexity of $\phi$,

$$
\frac{1+\rho_{\phi}\left(k^{1 / s} T_{\phi, u, A} f\right)}{k} \geqslant \frac{\rho_{\phi}\left(k^{1 / s} T_{\phi, u, A} f\right)}{k} \geqslant \rho_{\phi}\left(T_{\phi, u, A} f\right)
$$

By the proof of Lemma 3.1, $\rho_{\phi}\left(T_{\phi, u, A} f\right) \geqslant \phi(u \delta / 2)$. Hence there exists $u_{\varepsilon}>0$ such that for any $k>0, f \in B_{\phi},|f|_{\phi}^{s}<1-\delta$

$$
\frac{1+\rho_{\phi}\left(k^{1 / s} T_{\phi, u, A} f\right)}{k} \geqslant 1
$$

and consequently, $\left|T_{\phi, u_{\varepsilon}, A} f\right|_{\phi}^{s}>1-\varepsilon$.

THEOREM 3.3. Let $S_{\phi}$ denote the unit sphere in $E_{\phi}$ with respect to the Luxemburg $s$-convex norm $\|\cdot\|_{\phi}^{s}$. For any $\varepsilon>0$ there exists a retraction $R_{\phi}: B_{\phi} \rightarrow S_{\phi}$, such that for any $B \subset B_{\phi} \cap L_{1}\left(B_{k}\right)$

$$
\omega_{\phi}^{s}\left(R_{\phi}(B)\right) \leqslant(1+\varepsilon) \omega_{\phi}^{s}(B) .
$$

In particular, $k_{\omega_{\phi}^{s}}\left(E_{\phi}\right) \leqslant 1$.

Proof: Fix $\varepsilon>0$. Choose $\varepsilon_{1}>0$ such that $\left(1 /\left(1-\varepsilon_{1}\right)\right)^{s}<1+\varepsilon$. Let $u_{1}>0$ be a positive number corresponding to $\varepsilon_{1}$ by Lemma 3.1. Set for $f \in B_{\phi}$

$$
R_{\phi} f=\frac{T_{\phi, u_{1}} f}{\left(\left\|T_{\phi, u_{1}} f\right\|_{\phi}^{s}\right)^{1 / s}} .
$$


Observe that $\left\|R_{\phi} f\right\|_{\phi}^{s}=1$ for any $f \in B_{\phi}$. Moreover, by (3.1) and (3.3), $R_{\phi} f=f$ for any $f \in S_{\phi}$. By Corollary 2.5 and (3.3), $R_{\phi}$ is a continuous mapping. Now fix $B \subset B_{\phi} \cap L_{1}\left(B_{k}\right)$. By the choice of $u_{1}$ and Lemma 3.1, for any $f \in B$ and $h>0$,

$$
\begin{aligned}
\left\|R_{\phi} f-\left(R_{\phi} f\right)_{h}\right\|_{\phi}^{s} & =\left(\left\|T_{\phi, u_{1}} f-\left(T_{\phi, u_{1}} f\right)_{h}\right\|_{\phi}^{s}\right) /\left(\left\|T_{\phi, u_{1}} f\right\|_{\phi}^{s}\right) \\
& \leqslant\left(\left\|T_{\phi, u_{1}} f-\left(T_{\phi, u_{1}} f\right)_{h}\right\|_{\phi}^{s}\right) /\left(1-\varepsilon_{1}\right) .
\end{aligned}
$$

Consequently, by (3.7),

$$
\begin{aligned}
\omega_{\phi}^{s}\left(R_{\phi}(B)\right) & \leqslant \omega_{\phi}^{s}\left(\frac{T_{\phi, u_{1}}(B)}{1-\varepsilon_{1}}\right)=\frac{1}{\left(1-\varepsilon_{1}\right)^{s}} \omega_{\phi}^{s}\left(T_{\phi, u_{1}}(B)\right) \\
& \leqslant(1+\varepsilon) \omega_{\phi}^{s}\left(T_{\phi, u_{1}}(B)\right) \leqslant(1+\varepsilon) \omega_{\phi}^{s}(B) .
\end{aligned}
$$

The proof is complete.

Replacing Lemma 3.1 by Lemma 3.2 and reasoning as in Theorem 3.3, one can show

THEOREM 3.4. Let $S_{\phi, A}$ denote the unit sphere in $E_{\phi}$ with respect to the Amemiya $s$-convex norm $|\cdot|_{\phi}^{s}$. For any $\varepsilon>0$ there exists a retraction $R_{\phi, A}: B_{\phi, A}$ $\rightarrow S_{\phi, A}$, such that for any $B \subset B_{\phi, A} \cap L_{1}\left(B_{k}\right)$

$$
\omega_{\phi, A}^{s}\left(R_{\phi}(B)\right) \leqslant(1+\varepsilon) \omega_{\phi, A}^{s}(B) .
$$

In particular, $k_{\omega_{\phi, A}^{s}}\left(E_{\phi}\right) \leqslant 1$.

Remark 3.5. If $\phi$ is a convex function, then, by Remark 2.7, Theorem 3.3 (Theorem 3.4 respectively) holds true for any $B \subset B_{\phi}\left(B \subset B_{\phi, A}\right.$ respectively). If in the definition of $\omega_{\phi}^{s}$ for any $r>1$, we replace $\|\cdot\|_{\phi, \mathbb{R}^{k}}^{s}$ by $\|\cdot\|_{\phi, B_{k}(r)}^{s}$, where $\|\cdot\|_{\phi, B_{k}(r)}^{s}$, is the Luxemburg $s$-norm associated with a modular $\rho_{\phi, B_{k}(r)}(f)=\int_{B_{k}(r)} \phi(|f(t)|) d \mu_{k}(t)$ Theorem 3.3 remains true. In the case of the Amemiya norm the same applies to Theorem 3.4.

If $\phi$ is a convex function, by Remark 2.7, Proposition 2.9, Theorems 3.3 and 3.4, we easily get.

THEOREM 3.6. Let $\phi$ be a convex function such that $\phi^{*}(v)$ (see (2.6)) is finite for any $v>0$. Then for any $\varepsilon>0$ there exists a retraction $R_{\phi}: B_{\phi} \rightarrow S_{\phi}$ such that for any $B \subset B_{\phi}$

$$
\gamma_{\phi}^{1}\left(R_{\phi}(B)\right) \leqslant(2+\varepsilon) \gamma_{\phi}^{1}(B) .
$$

In particular, $k_{\gamma_{\phi}^{1}}\left(E_{\phi}\right) \leqslant 2$. The same result holds true in the case of the Amemiya norm.

REMARK 3.7. In the case of $\phi(t)=t^{p}(1 \leqslant p<\infty)$ and $k=1$, that is, $E_{\phi}=L_{p}[-1,1]$, Theorem 3.6 has been proven in [9, Corollary 6]. 


\section{REFERENCES}

[1] R.R. Akhmerov, M.I. Kamenskii, A.S. Potapov, A.S. Rodkina and B.N. Sadovskï, Measures of noncompactness and condensing operators (Birkhäuser, Basel, Boston, Berlin, 1992).

[2] Y. Benyamini and Y. Sternfeld, 'Spheres in infinite dimensional normed spaces are Lipschitz contractible', Proc. Amer. Math. Soc. 88 (1993), 439-445.

[3] K. Goebel and W.A. Kirk, Topics in metric fixed point theory (Cambridge University Press, Cambridge, 1990).

[4] A. Kufner, J. Oldr̆ich and F. Svatopulk, Function spaces (Noordhoff International Publishing, Prague, 1977).

[5] J. Musielak, Orlicz spaces and modular Spaces, Lecture Notes in Mathematics 1034 (Springer-Verlag, Berlin, Heidelberg, New York, 1983).

[6] B. Nowak, 'On the Lipschitzian of the unit ball in infinite dimensional Banach spaces onto its boundary', Bull. Acad. Polon. Sci. Sér. Sci. Math. 27 (1979), 861-864.

[7] A. Otáhal, 'Measure of noncompactness of subsets of Lebesgue spaces', Casopis Pest. Mat. 103 (1978), 69-72.

[8] J.M. Toledano, T.D. Benavides and G.L. Acedo, Measure of noncompactness in metric fixed point theory (Birkhäuser, Basel, Boston, Berlin, 1997).

[9] A. Trombetta and G. Trombetta, 'On the existence of $(\gamma)_{p} k$-set contractive retractions in $L_{p}[0,1]$ spaces, $1 \leqslant p<\infty$ ', Sci. Math. Jpn. 56 (2002), 327-335.

[10] J. Wośko, 'An example related to the retraction problem', Ann. Univ. Mariae Curie-Sktodowska 45 (1991), 127-130.

Department of Mathematics

Jagiellonian University

30-059 Kraków

Reymonta 4

Poland

e-mail: lewicki@im.uj.edu.pl
Department of Mathematics

University of Calabria

87036 Arcavacata di Rende

Cosenza

Italy

e-mail: trombetta@unical.it 